\title{
CENTRAL LIMIT THEOREM FOR A CLASS OF SPDES
}

\author{
PARISA FATHEDDIN, ${ }^{*}$ The University of Tennessee
}

\begin{abstract}
In this paper we establish the central limit theorem for a class of stochastic partial differential equations and as an application derive this theorem for two widely studied population models: super-Brownian motion and the Fleming-Viot process.

Keywords: Central limit theorem; stochastic partial differential equation; Fleming-Viot process; super-Brownian motion

2010 Mathematics Subject Classification: Primary 60F05
\end{abstract}

Secondary $60 \mathrm{H} 15 ; 60 \mathrm{~J} 68$

\section{Introduction}

Two commonly studied population models are super-Brownian motion (SBM) and the Fleming-Viot process (FVP). These are measure-valued Markov processes and can be represented as stochastic partial differential equations (SPDEs). We use these representations to formulate a general class of SPDE and investigate the central limit theorem (CLT) for this class and study the two population models as special cases. The large and moderate deviation principles for this class of SPDEs and the two population models were established in [4] and [5], respectively.

SBM is the continuous version of the branching Brownian motion, the oldest and best-known branching process, where individuals are assumed to reproduce following a Galton-Watson process. In this model, the population evolves as a 'cloud' through time with each individual assumed to move according to a Brownian motion, leaving behind a random number of offspring upon its death. On the other hand, the FVP is the continuous approximation of a step-wise mutation process, in which each individual has a 'type' (usually a genetic type) given by an element $x$ in some set $E$. In this model, we are interested in the distributions of the types in the whole population making the FVP a probability measure-valued process. Mutation is the term referring to a change in genetic type. In the FVP the number of individuals is assumed to be fixed throughout time; that is, in the place of an individual's death an offspring is born. For more information and background on SBM and the FVP and their formulation as the continuous approximation of discrete particle systems, we refer the reader to [3].

On the topic of the CLT, developments have been made on SBM and on various processes related to it. $\mathrm{Li}$ [16] considered the critical continuous SBM and proved the CLT in all dimensions $d \geq 1$ and also derived the CLT for its weighted occupation time process in $d \geq 3$. Schied [18] showed the tightness and weak convergence of the finite-dimensional distributions of SBM to those of a Wiener process as part of the proof of the moderate deviation principle for SBM for $d \geq 1$. The CLT for SBM was then concluded for all dimensions. In [15], Lee and Remillard also used their large and moderate deviation results to derive

Received 24 April 2014; revision received 30 July 2014.

* Current address: Air Force Institute of Technology, 2950 Hobson Way, WPAFB, OH 45433-776, USA.

Email address: graduate24@gmail.com 
the CLT for SBM in dimension $d=3$. In addition, some authors have studied SBM with super-Brownian immigration (SBMSBI). Hong and Li [10] proved the CLT for SBMSBI for dimensions $d \geq 3$ and later Hong [9] showed the CLT under the quenched probability law for the same dimensions. Hong and Zeitouni [13] also succeeded in achieving the quenched CLT for SBMSBI for dimensions $d \geq 4$. In addition, Hong [8] showed the CLT for the occupation time process of SBMSBI for $d \geq 3$. The CLT for SBM with other types of immigration has also been considered. See, for example, [7], [11], [12], and [22]. To the best of the author's knowledge, the CLT has not been shown for the FVP.

Most of the authors mentioned above proved the CLT by using the Laplace transform of the process under study. However, Schied [18], Lee and Remillard [15], and Zhang [22] applied the method offered by Iscoe [14] to achieve the CLT. To be more precise, Iscoe's method consists of finding the limit of the Laplace functional of the centered process and then applying the Bochner-Minlos theorem. That is, to achieve the CLT for a process $\left\{X_{t}\right\}$ first the centered functional process is formed by

$$
\left\langle Z_{t}, f\right\rangle=a_{d}^{-1}(t)\left(\left\langle X_{t}, f\right\rangle-\mathbb{E}\left\langle X_{t}, f\right\rangle\right)
$$

for some norming constant $a_{d}(t)$ and for $f \in \delta(\mathbb{R})$, the Schwartz space. The expectation is denoted by $\mathbb{E}$. Then the weak convergence of $Z_{t}$ to a centered Gaussian process $Z_{\infty}$ is obtained by considering the Laplace functional of the centered functional process

$$
\mathbb{E} \exp \left(-\left\langle Z_{t}, f\right\rangle\right)=\exp \left(W_{t}\right)
$$

where the limit of $W_{t}$ is the covariance of the Gaussian process $Z_{\infty}$.

Here, instead of the Laplace transform of the SBM, we use another characterization of this population model given by Xiong [20]. In [20], by studying SBM as a 'distribution' functionvalued process, an SPDE was formed to define the SBM. A similar SPDE was also derived for the FVP. By observing the similarities between the two SPDEs, we formulate a general SPDE and derive the CLT in dimension $d=1$ for this SPDE, and as an application establish the CLT for the two population models. We note that since the formulation of the general SPDE and the two population models given in [20] is in $d=1$ only, our results are limited to this dimension. Extending the result of [20] and also the result presented here to higher dimensions requires further investigation.

In Section 2 we begin with some background and then introduce the notation used in this paper. We then prove the CLT for the general SPDE in Section 3 by first showing its tightness in our space, introduced in Section 2, and afterwards prove that the limiting process has a unique solution and is Gaussian. Section 4 contains the CLT for the two population models; SBM and the FVP.

\section{Notation and main results}

Suppose $(\Omega, \mathcal{F}, \mathbb{P})$ is a probability space and $\left\{\mathcal{F}_{t}\right\}$ is a family of nondecreasing rightcontinuous sub- $\sigma$-fields of $\mathcal{F}$ such that $\mathcal{F}_{0}$ contains all $\mathbb{P}$-null subsets of $\Omega$. We denote $\mathcal{C}_{\mathrm{b}}(\mathbb{R})$ to be the space of continuous bounded functions on $\mathbb{R}$ and $\mathcal{C}_{\mathrm{c}}(\mathbb{R})$ to be composed of continuous functions in $\mathbb{R}$ with compact support. Let $K$ be a constant that may change values at different lines. Let

$$
\mathcal{C}_{p}\left(\mathbb{R}^{d}\right):=\left\{f \in \mathcal{C}\left(\mathbb{R}^{d}\right): \sup \frac{|f(x)|}{\phi_{p}(x)}<\infty \text { for } p>d, \phi_{p}(x):=\left(1+|x|^{2}\right)^{-p / 2}\right\} .
$$


Since SBM is a measure-valued process, we denote it by $\mu_{t}^{\varepsilon}$ with branching rate $\varepsilon$. There are two common ways to define SBM, $\mu_{t}^{\varepsilon}$. One is by its Laplace transform, given by

$$
\mathbb{E}_{\mu_{0}^{\varepsilon}} \exp \left(-\left\langle\mu_{t}^{\varepsilon}, f\right\rangle\right)=\exp \left(-\left\langle\mu_{0}^{\varepsilon}, v(t, \cdot)\right\rangle\right)
$$

where $v(\cdot, \cdot)$ is the unique mild solution of the evolution equation

$$
\dot{v}(t, x)=\frac{1}{2} \Delta v(t, x)-v^{2}(t, x), \quad v(0, x)=f(x) \quad \text { for } f \in \mathcal{C}_{p}^{+}\left(\mathbb{R}^{d}\right) .
$$

The other is as the unique solution to a martingale problem. For all $f \in \mathcal{C}_{\mathrm{b}}^{2},(\mathbb{R})$,

$$
M_{t}(f):=\left\langle\mu_{t}^{\varepsilon}, f\right\rangle-\left\langle\mu_{0}^{\varepsilon}, f\right\rangle-\int_{0}^{t}\left\langle\mu_{s}^{\varepsilon}, \frac{1}{2} \Delta f\right\rangle \mathrm{d} s
$$

is a square-integrable martingale with quadratic variation

$$
\langle M(f)\rangle_{t}=\varepsilon \int_{0}^{t}\left\langle\mu_{s}^{\varepsilon}, f^{2}\right\rangle \mathrm{d} s .
$$

Similarly, let $\mu_{t}^{\varepsilon}$ denote the FVP with mutation rate $\varepsilon$. Here, $\left\{\mu_{t}^{\varepsilon}\right\}$ is a family of probability measures. There are two usual methods of defining this process. The first is as a Markov process with generator

$$
\begin{aligned}
\mathcal{L}^{\varepsilon} F\left(\mu_{t}^{\varepsilon}\right)= & f^{\prime}\left(\left\langle\mu_{t}^{\varepsilon}, \phi\right\rangle\right)\left\langle\mu_{t}^{\varepsilon}, A \phi\right\rangle \\
& +\frac{\varepsilon}{2} \iint f^{\prime \prime}\left(\left\langle\mu_{t}^{\varepsilon}, \phi\right\rangle\right) \phi(x) \phi(y) Q\left(\mu_{t} ; \mathrm{d} x, \mathrm{~d} y\right),
\end{aligned}
$$

which has domain

$$
\mathscr{D}=\left\{F: F\left(\mu_{t}^{\varepsilon}\right)=f\left(\left\langle\mu_{t}^{\varepsilon}, \phi\right\rangle\right), f \in \mathcal{C}_{\mathrm{b}}^{\infty}(\mathbb{R}), \phi \in D(A), \mu \in M_{1}(E)\right\},
$$

where $\mathcal{C}_{\mathrm{b}}^{\infty}(\mathbb{R})$ is the set of all bounded, infinitely differentiable functions on $\mathbb{R}$ and $M_{1}(E)$ is the space of all probability measures on $E$ endowed with the usual weak topology. Furthermore, $D(A)$ denotes the domain of $A$, where $A$ is the generator of a Markov process on the set $E=[0,1]$. In the context of population models, $E$ represents the genetic-type space of the population and $A$ is referred to as the mutation operator. Moreover,

$$
Q\left(\mu_{t}^{\varepsilon} ; \mathrm{d} x, \mathrm{~d} y\right)=\mu_{t}^{\varepsilon}(\mathrm{d} x) \delta_{x}(\mathrm{~d} y)-\mu_{t}^{\varepsilon}(\mathrm{d} x) \mu_{t}^{\varepsilon}(\mathrm{d} y),
$$

where $\delta_{x}$ denotes the Dirac measure at $x$. For more information on this characterization of the FVP, see [2] and [6].

The second method to define the FVP is as a unique solution to a martingale problem. For $f \in \mathcal{C}_{\mathrm{c}}^{2}(\mathbb{R})$,

$$
M_{t}(f)=\left\langle\mu_{t}^{\varepsilon}, f\right\rangle-\left\langle\mu_{0}^{\varepsilon}, f\right\rangle-\int_{0}^{t}\left\langle\mu_{s}^{\varepsilon}, \frac{1}{2} \Delta f\right\rangle \mathrm{d} s
$$

is a continuous square-integrable martingale with quadratic variation

$$
\langle M(f)\rangle_{t}=\varepsilon \int_{0}^{t}\left(\left\langle\mu_{s}^{\varepsilon}, f^{2}\right\rangle-\left\langle\mu_{s}^{\varepsilon}, f\right\rangle^{2}\right) \mathrm{d} s .
$$


Recently, another formulation of SBM and the FVP was given in [20] by considering a 'distribution' function-valued process. More precisely, by considering $u_{t}^{\varepsilon}(y)=\int_{0}^{y} \mu_{t}^{\varepsilon}(\mathrm{d} x)$ for all $y \in \mathbb{R}$, the SBM was characterized in [20] by the following SPDE:

$$
u_{t}^{\varepsilon}(y)=F(y)+\int_{0}^{t} \int_{0}^{u_{s}^{\varepsilon}(y)} W(\mathrm{~d} s \mathrm{~d} a)+\int_{0}^{t} \frac{1}{2} \Delta u_{s}^{\varepsilon}(y) \mathrm{d} s,
$$

where $F(y)=\int_{0}^{y} \mu_{0}^{\varepsilon}(\mathrm{d} x)$ and $W$ is a white noise random measure on $\mathbb{R}^{+} \times \mathbb{R}$ with intensity measure $\mathrm{d} s \mathrm{~d} a$.

Also, by considering $u_{t}^{\varepsilon}(y)=\int_{-\infty}^{y} \mu_{t}^{\varepsilon}(\mathrm{d} x)$ for all $y \in \mathbb{R}$, the FVP was given in [20] by the following SPDE:

$$
u_{t}^{\varepsilon}(y)=F(y)+\int_{0}^{t} \int_{0}^{1}\left(\mathbf{1}_{\left\{a \leq u_{s}^{\varepsilon}(y)\right\}}-u_{s}^{\varepsilon}(y)\right) W(\mathrm{~d} s \mathrm{~d} a)+\int_{0}^{t} \frac{1}{2} \Delta u_{s}^{\varepsilon}(y) \mathrm{d} s,
$$

where $\mathbf{1}$ is the indicator function. We denote both processes as $\mu_{t}^{\varepsilon}$, where based on the context it will be clear which process is being referred to. By noting the similarities between the two SPDE formulations given above, we form a general SPDE with the two models as special classes as

$$
u_{t}^{\varepsilon}(y)=F(y)+\sqrt{\varepsilon} \int_{0}^{t} \int_{U} G\left(a, y, u_{s}^{\varepsilon}(y)\right) W(\mathrm{~d} s \mathrm{~d} a)+\int_{0}^{t} \frac{1}{2} \Delta u_{s}^{\varepsilon}(y) \mathrm{d} s,
$$

where $G: U \times \mathbb{R}^{2} \rightarrow \mathbb{R}, F$ is a function on $\mathbb{R}$ and for $u_{1}, u_{2}, u, y \in \mathbb{R}$,

$$
\begin{gathered}
\int_{U}\left|G\left(a, y, u_{1}\right)-G\left(a, y, u_{2}\right)\right|^{2} \lambda(\mathrm{d} a) \leq K\left|u_{1}-u_{2}\right|, \\
\int_{U}|G(a, y, u)|^{2} \lambda(\mathrm{d} a) \leq K\left(1+|u|^{2}\right) .
\end{gathered}
$$

Let $\delta(\mathbb{R})$ be the Schwartz space of rapidly decreasing functions defined as

$$
\S(\mathbb{R})=\left\{\phi \in \mathcal{C}^{\infty}(\mathbb{R}):\|\phi\|_{\alpha, \beta}<\infty, \text { for all } \alpha, \beta \in \mathbb{N} \cup\{0\}\right\},
$$

where $\|\phi\|_{\alpha, \beta}=\sup _{x \in \mathbb{R}}\left|x^{\alpha} \phi^{(\beta)}(x)\right|$ with its dual $\delta^{\prime}(\mathbb{R})$ known as the space of tempered distributions. To investigate the CLT for the general SPDE, we consider the $f^{\prime}(\mathbb{R})$-valued centered process

$$
Z_{t}^{\varepsilon}=\frac{1}{\sqrt{\varepsilon}}\left(u_{t}^{\varepsilon}-u_{t}^{0}\right)
$$

Namely, we study the following process:

$$
\begin{aligned}
\left\langle Z_{t}^{\varepsilon}, f\right\rangle:= & \int_{0}^{t} \int_{U} \int_{\mathbb{R}} G\left(a, y, u_{s}^{\varepsilon}(y)\right) f(y) \mathrm{d} y W(\mathrm{~d} a \mathrm{~d} s) \\
& +\frac{1}{2} \int_{0}^{t}\left\langle Z_{s}^{\varepsilon}, f^{\prime \prime}\right\rangle \mathrm{d} s \quad \text { for } f \in \&(\mathbb{R}) .
\end{aligned}
$$

Theorem 1. The centered process $\left\{Z_{t}^{\varepsilon}\right\}$ is tight in $\mathcal{C}\left([0,1] ; f^{\prime}(\mathbb{R})\right)$.

We use the above theorem to obtain the following results on the CLT. 
Theorem 2. The general SPDE $\left\{u_{t}^{\varepsilon}\right\}$ satisfies the CLT in space $\mathcal{C}\left([0,1], \mathcal{S}^{\prime}(\mathbb{R})\right)$, where $\left\{Z_{t}^{\varepsilon}\right\}$ converges in distribution as $\varepsilon$ tends to 0 to a Gaussian process $\left\{Z_{t}^{0}\right\}$ with 0 mean and covariance

$$
\begin{aligned}
\operatorname{cov}\left(\left\langle Z_{t}^{0}, f\right\rangle,\left\langle Z_{t}^{0}, g\right\rangle\right)= & \int_{0}^{t} \int_{U} \int_{\mathbb{R}} G\left(a, y, u_{s}^{0}(y)\right) f(y) \mathrm{d} y \\
& \times \int_{\mathbb{R}} G\left(a, x, u_{s}^{0}(x)\right) g(x) \mathrm{d} x \lambda(\mathrm{d} a) \mathrm{d} s \quad \text { for } f, g \in \delta(\mathbb{R}) .
\end{aligned}
$$

For the next two theorems, let $\mu_{t}^{\varepsilon}$ denote the SBM and the FVP, and consider the centered process

$$
\tilde{Z}_{t}^{\varepsilon}=\frac{1}{\sqrt{\varepsilon}}\left(\mu_{t}^{\varepsilon}-\mu_{t}^{0}\right) .
$$

Theorem 3. The SBM satisfies the CLT in space $\mathcal{C}\left([0,1], \mathcal{f}^{\prime}(\mathbb{R})\right)$, where $\left\{\tilde{Z}_{t}^{\varepsilon}\right\}$ converges in distribution as $\varepsilon$ tends to 0 to a Gaussian process, $\left\{\tilde{Z}_{t}^{0}\right\}$ with 0 mean and covariance

$$
\operatorname{cov}\left(\left\langle\tilde{Z}_{t}^{\varepsilon}, f\right\rangle,\left\langle\tilde{Z}_{t}^{\varepsilon}, g\right\rangle\right)=\int_{0}^{t}\left\langle\mu_{s}^{0}, f g\right\rangle \mathrm{d} s \quad \text { for } f, g \in \mathcal{R}(\mathbb{R}) .
$$

Theorem 4. The FVP satisfies the CLT in space $\mathcal{C}\left([0,1], \mathcal{f}^{\prime}(\mathbb{R})\right)$, where $\left\{\tilde{Z}_{t}^{\varepsilon}\right\}$ converges in distribution as $\varepsilon$ tends to 0 to a Gaussian process, $\left\{\tilde{Z}_{t}^{0}\right\}$ with 0 mean and covariance

$$
\begin{aligned}
\operatorname{cov}\left(\left\langle\tilde{Z}_{t}^{\varepsilon}, f\right\rangle,\left\langle\tilde{Z}_{t}^{\varepsilon}, g\right\rangle\right)= & \int_{0}^{t} \int_{0}^{1} f(y) g(y) \mu_{s}^{0}(\mathrm{~d} y) \mathrm{d} s-\int_{0}^{t} \int_{0}^{1}\left\langle\mu_{s}^{0}, f\right\rangle g(y) \mu_{s}^{0}(\mathrm{~d} y) \mathrm{d} s \\
& -\int_{0}^{t} \int_{0}^{1} f(y)\left\langle\mu_{s}^{0}, g\right\rangle \mu_{s}^{0}(\mathrm{~d} y) \mathrm{d} s \\
& +\int_{0}^{t}\left\langle\mu_{s}^{0}, f\right\rangle\left\langle\mu_{s}^{0}, g\right\rangle \mathrm{d} s \quad \text { for } f, g \in \delta(\mathbb{R}) .
\end{aligned}
$$

\section{The CLT for the general SPDE}

\subsection{Proof of Theorem 1}

Since the strong uniqueness of solutions to the general SPDE $\left\{u_{t}^{\varepsilon}\right\}$ was obtained in [20], then there exists a unique solution to $Z_{t}^{\varepsilon}$; consequently, we have the uniqueness of solutions to our process of study $\left\langle Z_{t}^{\varepsilon}, f\right\rangle$. Thus, we use its mild solution instead, given by

$$
\left\langle Z_{t}^{\varepsilon}, f\right\rangle=\int_{0}^{t} \int_{U} \int_{\mathbb{R}} P_{t-s} G\left(a, y, u_{s}^{\varepsilon}(y)\right) f(y) \mathrm{d} y W(\mathrm{~d} a \mathrm{~d} s),
$$

where $P_{t-s}$ is the Brownian semigroup defined as $P_{t} f(x)=\int_{\mathbb{R}} p_{t}(x-y) f(y) \mathrm{d} y$ with

$$
p_{t}(x-y)=\frac{1}{\sqrt{2 \pi t}} \exp \left(-\frac{|x-y|^{2}}{2 t}\right)
$$

being the heat kernel. We show that $\left\langle Z_{t}^{\varepsilon}, f\right\rangle$ is tight in $\mathcal{C}\left([0,1] ; \mathcal{\prime}^{\prime}(\mathbb{R})\right)$ by applying a classic result given below.

Lemma 1. (See $\left[1\right.$, Theorem 12.3].) The sequence $\left\{X_{n}\right\}$ is tight in $\mathcal{C}([0,1] ; \mathbb{R})$ if it satisfies the following two conditions:

(i) the sequence $\left\{X_{n}(0)\right\}$ is tight; 
(ii) there exist constants $\gamma \geq 0$ and $\alpha>1$ and a nondecreasing, continuous function $F$ on $[0,1]$ such that

$$
\mathbb{P}\left(\left|X_{n}\left(t_{2}\right)-X_{n}\left(t_{1}\right)\right| \geq \lambda\right) \leq \frac{1}{\lambda \gamma}\left|F\left(t_{2}\right)-F\left(t_{1}\right)\right|^{\alpha}
$$

holds for all $t_{1}, t_{2}$, and $n$ and all positive $\lambda$.

As stated in [1], the moment condition

$$
\mathbb{E}\left(\left|X_{n}\left(t_{2}\right)-X_{n}\left(t_{1}\right)\right|^{\gamma}\right) \leq\left|F\left(t_{2}\right)-F\left(t_{1}\right)\right|^{\alpha}
$$

implies (7). Note that in our case, $\left\{X_{n}(0)\right\}=0$ for all $n$. Therefore, it is sufficient to prove Lemma 1(ii) by checking that $\left\langle Z_{t}^{\varepsilon}, f\right\rangle$ satisfies the moment condition given by (8). To this end, we use the following lemma, the proof of which is very similar to the proof of Lemma 2.3 in [20].

Lemma 2. For any $n \geq 2$,

$$
\mathbb{E}\left(\sup _{\varepsilon>0} \sup _{0 \leq s \leq 1} \int_{\mathbb{R}}\left|u_{s}^{\varepsilon}(x)\right|^{2} \mathrm{e}^{-2|x|} \mathrm{d} x\right)^{n}<\infty .
$$

Given any $t_{1}, t_{2} \in[0,1]$ and without loss of generality, we assume that $t_{1}<t_{2}$ and with the help of the Burkholder-Davis-Gundy inequality and (4), we obtain

$$
\begin{aligned}
\mathbb{E}\left|\left\langle Z_{t_{2}}^{\varepsilon}, f\right\rangle-\left\langle Z_{t_{1}}^{\varepsilon}, f\right\rangle\right|^{4} \\
\quad=\mathbb{E}\left|\int_{t_{1}}^{t_{2}} \int_{U} \int_{\mathbb{R}} P_{t-s} G\left(a, y, u_{s}^{\varepsilon}(y)\right) f(y) \mathrm{d} y W(\mathrm{~d} a \mathrm{~d} s)\right|^{4} \\
\quad \leq \mathbb{E}\left(\int_{t_{1}}^{t_{2}} \int_{U}\left(\int_{\mathbb{R}} P_{t-s} G\left(a, y, u_{s}^{\varepsilon}(y)\right) f(y) \mathrm{d} y\right)^{2} \lambda(\mathrm{d} a) \mathrm{d} s\right)^{2} \\
\quad \leq \mathbb{E}\left(\int_{t_{1}}^{t_{2}} \int_{U} \int_{\mathbb{R}} G\left(a, y, u_{s}^{\varepsilon}(y)\right)^{2} \mathrm{e}^{-2|\lfloor y\rfloor|} \mathrm{d} y \int_{\mathbb{R}}\left(P_{t-s} f(r)\right)^{2} \mathrm{e}^{2|\lfloor r\rfloor|} \mathrm{d} r \lambda(\mathrm{d} a) \mathrm{d} s\right)^{2} \\
\quad \leq \mathbb{E}\left(\int_{t_{1}}^{t_{2}} \int_{\mathbb{R}} K\left(1+\left|u_{s}^{\varepsilon}(y)\right|^{2}\right) \mathrm{e}^{-2|\lfloor y\rfloor|} \mathrm{d} y \int_{\mathbb{R}}\left(P_{t-s} f(r)\right)^{2} \mathrm{e}^{2|\lfloor r\rfloor|} \mathrm{d} r \mathrm{~d} s\right)^{2},
\end{aligned}
$$

where $\lfloor y\rfloor$ is the greatest integer less than or equal to $y$. Using the fact that for any $f \in \delta\left(\mathbb{R}^{d}\right)$, $\left\|P_{s} f\right\| \leq K\left(1 \wedge s^{-d / 2}\right)$, we obtain

$$
\begin{aligned}
& \int_{\mathbb{R}}\left(P_{t-s} f(y)\right)^{2} \mathrm{e}^{2|\lfloor y\rfloor|} \mathrm{d} y \\
& \quad \leq K\left(1 \wedge(t-s)^{-1 / 2}\right) \int_{\mathbb{R}} P_{t-s} f(y) \mathrm{e}^{2|\lfloor y\rfloor|} \mathrm{d} y \\
& \quad \leq K\left(1 \wedge(t-s)^{-1 / 2}\right) \int_{\mathbb{R}} \int_{\mathbb{R}} \exp \left(-\frac{|x|^{2}}{2(t-s)}\right)\left(\exp \left(\left\lceil\frac{2|x y|}{2(t-s)}\right\rceil\right) f(x)\right) \\
& \quad \times \exp \left(-\frac{|y|^{2}}{2(t-s)}+2|\lfloor y\rfloor|\right) \mathrm{d} x \mathrm{~d} y \\
& \leq K,
\end{aligned}
$$


where $\lceil y\rceil$ is the least integer greater than or equal to $y$. Hence, by Lemma 2,

$$
\mathbb{E}\left|\left\langle Z_{t_{1}}^{\varepsilon}, f\right\rangle-\left\langle Z_{t_{2}}^{\varepsilon}, f\right\rangle\right|^{4} \leq K\left|t_{1}-t_{2}\right|^{2} .
$$

Therefore, $\left\{\left\langle Z_{t}^{\varepsilon}, f\right\rangle\right\}$ is tight in $\mathcal{C}([0,1] ; \mathbb{R})$. Based on [17, Theorem 3.1], we conclude the tightness of $\left\{\left\langle Z_{t}^{\varepsilon}, f\right\rangle\right\}$ in $\mathcal{C}\left([0,1] ; \boldsymbol{\wp}^{\prime}(\mathbb{R})\right)$.

\subsection{Proof of Theorem 2}

We prove the convergence of the centered process of the general SPDE to a Gaussian process to obtain the CLT for the general SPDE. We can achieve the $L^{2}$-convergence of $\left\{\left\langle Z_{t}^{\varepsilon}, f\right\rangle\right\}$ as $\varepsilon \rightarrow 0$ using the Burkholder-Davis-Gundy inequality and (3) of $G\left(a, \cdot, u_{s}^{\varepsilon}().\right)$ as

$$
\begin{aligned}
\mathbb{E}\left|\left\langle Z_{t}^{\varepsilon}, f\right\rangle-\left\langle Z_{t}^{0}, f\right\rangle\right|^{2} \\
\quad=\mathbb{E}\left|\int_{0}^{t} \int_{U} \int_{\mathbb{R}} P_{t-s}\left(G\left(a, y, u_{s}^{\varepsilon}(y)\right)-G\left(a, y, u_{s}^{0}(y)\right)\right) f(y) \mathrm{d} y W(\mathrm{~d} a \mathrm{~d} s)\right|^{2} \\
\leq \mathbb{E}\left|\int_{0}^{t} \int_{U}\left(\int_{\mathbb{R}} P_{t-s}\left(G\left(a, y, u_{s}^{\varepsilon}(y)\right)-G\left(a, y, u_{s}^{0}(y)\right)\right) f(y) \mathrm{d} y\right)^{2} \lambda(\mathrm{d} a) \mathrm{d} s\right| \\
\leq K \mathbb{E}\left|\int_{0}^{t} \int_{U} \int_{\mathbb{R}}\right| G\left(a, y, u_{s}^{\varepsilon}(y)\right)-\left.G\left(a, y, u_{s}^{0}(y)\right)\right|^{2} \mathrm{e}^{-2|\lfloor y\rfloor|} \mathrm{d} y \lambda(\mathrm{d} a) \mathrm{d} s \mid \\
\leq K \mathbb{E}\left|\int_{0}^{t} \int_{\mathbb{R}}\right| u_{s}^{\varepsilon}(y)-u_{s}^{0}(y)\left|\mathrm{e}^{-2|\lfloor y\rfloor|} \mathrm{d} y \mathrm{~d} s\right| \\
\leq K \mathbb{E}\left|\int_{0}^{t}\left(\int_{\mathbb{R}}\left|u_{s}^{\varepsilon}(y)-u_{s}^{0}(y)\right|^{2} \mathrm{e}^{-2|\lfloor y\rfloor|} \mathrm{d} y\right)^{1 / 2}\left(\int_{\mathbb{R}} \mathrm{e}^{-2|\llcorner y\rfloor|} \mathrm{d} y\right)^{1 / 2} \mathrm{~d} s\right| \\
\leq K \mathbb{E}\left|\int_{0}^{t}\left(\int_{\mathbb{R}}\left|u_{s}^{\varepsilon}(y)-u_{s}^{0}(y)\right|^{2} \mathrm{e}^{-2|\lfloor y\rfloor|} \mathrm{d} y\right)^{1 / 2} \mathrm{~d} s\right|,
\end{aligned}
$$

where we used (10) in the third step. By applying Jensen's inequality for concave functions, we can use Lemma 2 and the dominated convergence theorem to attain the convergence of $\mathbb{E}\left|\left\langle Z_{t}^{\varepsilon}, f\right\rangle-\left\langle Z_{t}^{0}, f\right\rangle\right|^{2} \rightarrow 0$ as $\varepsilon \rightarrow 0$. Thus, we obtain the convergence in distribution of $\left\langle Z_{t}^{\varepsilon}, f\right\rangle$ to

$$
\left\langle Z_{t}^{0}, f\right\rangle=\int_{0}^{t} \int_{U} \int_{\mathbb{R}} P_{t-s} G\left(a, y, u_{s}^{0}(y)\right) f(y) \mathrm{d} y W(\mathrm{~d} a \mathrm{~d} s) .
$$

The tightness result obtained above implies that $\left\{\left\langle Z_{t}^{\varepsilon}, f\right\rangle\right\}$ is relatively compact by Prohorov's theorem. We now apply the following tightness criterion stated in [19].

Lemma 3. (See [19, Corollary 11.6.1].) Let $\left\{\mathbb{P}_{n}\right\}_{n \geq 1}$ be a sequence of probability measures on a metric space $(S, m)$. If the sequence $\left\{\mathbb{P}_{n}\right\}$ is tight and the limit of any convergence subsequence from $\left\{\mathbb{P}_{n}\right\}$ must be $\mathbb{P}$, then $\mathbb{P}_{n} \stackrel{\mathrm{D}}{\rightarrow} \mathbb{P}$, where $\stackrel{\mathrm{D}}{\rightarrow}$ ' denotes convergence in distribution.

However, every subsequence in our case has the form given in (6) and the uniqueness of solutions to (12) can be derived analogous to estimates in (11). Since $u_{s}^{0}(y)$ is a partial differential equation then the integrand in the Itô integral (12) is deterministic and by applying the Hölder inequality and following similar steps to (9), we can show that

$$
\int_{0}^{1} \int_{U}\left(\int_{\mathbb{R}} P_{t-s} G\left(a, y, u_{s}^{0}(y)\right) f(y) \mathrm{d} y\right)^{2} \lambda(\mathrm{d} a) \mathrm{d} s<\infty,
$$


which implies that $Z_{t}^{0}$ is a Gaussian process with 0 mean and covariance

$$
\begin{aligned}
\operatorname{cov}( & \left.\left\langle Z_{t}^{0}, f\right\rangle,\left\langle Z_{t}^{0}, g\right\rangle\right) \\
& =\int_{0}^{t} \int_{U} \int_{\mathbb{R}} P_{t-s} G\left(a, y, u_{s}^{0}(y)\right) f(y) \mathrm{d} y \int_{\mathbb{R}} P_{t-s} G\left(a, r, u_{s}^{0}(r)\right) g(r) \mathrm{d} r \lambda(\mathrm{d} a) \mathrm{d} s .
\end{aligned}
$$

Note that because of the transition invariant property of the Lebesgue measure, (14) is equivalent to $(5)$.

\section{The CLT for the SBM and the FVP}

Now we turn our attention to the two population models mentioned in the introduction.

\subsection{Proof of Theorem 3}

For the $\operatorname{SBM}\left\{\mu_{t}^{\varepsilon}\right\}$ based on SPDE (1), we have $G(a, y, u)=\mathbf{1}_{\{0 \leq a \leq u\}}+\mathbf{1}_{\{u \leq a \leq 0\}}$, which satisfies (3) and (4). Therefore, the tightness result obtained for the general SPDE can be used in this case. As for the limit, recall that $u_{t}^{\varepsilon}(y)=\int_{0}^{y} \mu_{t}^{\varepsilon}(\mathrm{d} x)$ and, thus, for $f \in \mathcal{S}(\mathbb{R})$,

$$
\left\langle\mu_{t}^{\varepsilon}, f\right\rangle=-\left\langle u_{t}^{\varepsilon}, f^{\prime}\right\rangle .
$$

Then the centered functional process for SBM is found by

$$
\begin{aligned}
\left\langle\tilde{Z}_{t}^{\varepsilon}, f\right\rangle & =\left\langle\frac{1}{\sqrt{\varepsilon}}\left(\mu_{t}^{\varepsilon}-\mu_{t}^{0}\right), f\right\rangle \\
& =\left\langle\frac{1}{\sqrt{\varepsilon}}\left(-u_{t}^{\varepsilon}+u_{t}^{0}\right), f^{\prime}\right\rangle \\
& =-\left\langle Z_{t}^{\varepsilon}, f^{\prime}\right\rangle \\
& =-\int_{0}^{t} \int_{U} \int_{\mathbb{R}} P_{t-s} G\left(a, y, u_{s}^{\varepsilon}(y)\right) f^{\prime}(y) \mathrm{d} y W(\mathrm{~d} a \mathrm{~d} s) .
\end{aligned}
$$

Based on our estimates in the case of the general SPDE, the limit of (15) is

$$
\left\langle\tilde{Z}_{t}^{0}, f\right\rangle=-\int_{0}^{t} \int_{U} \int_{\mathbb{R}} P_{t-s} G\left(a, y, u_{s}^{0}(y)\right) f^{\prime}(y) \mathrm{d} y W(\mathrm{~d} a \mathrm{~d} s) .
$$

Namely, we have

$$
\begin{aligned}
\left\langle\tilde{Z}_{t}^{0}, f\right\rangle & \\
& =-\int_{0}^{t} \int_{\mathbb{R}} \int_{\mathbb{R}}\left(\mathbf{1}_{\left\{0 \leq a \leq u_{s}^{0}(y)\right\}}+\mathbf{1}_{\left\{u_{s}^{0}(y) \leq a \leq 0\right\}}\right) f^{\prime}(y) \mathrm{d} y W(\mathrm{~d} s \mathrm{~d} a) \\
& =-\int_{0}^{t} \int_{0}^{u_{s}^{0}(y)} \int_{0}^{\infty} f^{\prime}(y) \mathrm{d} y W(\mathrm{~d} a \mathrm{~d} s)-\int_{0}^{t} \int_{u_{s}^{0}(y)}^{0} \int_{-\infty}^{0} f^{\prime}(y) \mathrm{d} y W(\mathrm{~d} a \mathrm{~d} s) \\
& =-\int_{0}^{t} \int_{0}^{\infty} \int_{\left(u_{s}^{0}\right)^{-1}(a)}^{\infty} f^{\prime}(y) \mathrm{d} y W(\mathrm{~d} a \mathrm{~d} s)-\int_{0}^{t} \int_{-\infty}^{0} \int_{-\infty}^{\left(u_{s}^{0}\right)^{-1}(a)} f^{\prime}(y) \mathrm{d} y W(\mathrm{~d} a \mathrm{~d} s) \\
& =\int_{0}^{t} \int_{0}^{\infty} f\left(\left(u_{s}^{0}\right)^{-1}(a)\right) W(\mathrm{~d} a \mathrm{~d} s)-\int_{0}^{t} \int_{-\infty}^{0} f\left(\left(u_{s}^{0}\right)^{-1}(a)\right) W(\mathrm{~d} a \mathrm{~d} s) \\
& =: I_{t}^{1}(f)-I_{t}^{2}(f),
\end{aligned}
$$


where we have denoted $a=u_{s}^{0}(y)$ giving $\left(u_{s}^{0}\right)^{-1}(a)=y$. Then

$$
\operatorname{cov}\left(\left\langle\tilde{Z}_{t}^{0}, f\right\rangle,\left\langle\tilde{Z}_{t}^{0}, g\right\rangle\right)=\mathbb{E}\left(\left(I_{1}^{t}(f)-I_{2}^{t}(f)\right)\left(I_{1}^{t}(g)-I_{2}^{t}(g)\right)\right) .
$$

Because of the measurability of $f$ and $g$, we can write $f\left(\left(u_{s}^{0}\right)^{-1}(a)\right)$ and $g\left(\left(u_{s}^{0}\right)^{-1}(a)\right)$ as limits of simple functions, $\sum_{i} \alpha_{i} \mathbf{1}_{\left\{A_{i}\right\}}$ and $\sum_{j} \beta_{j} \mathbf{1}_{\left\{B_{j}\right\}}$, respectively. Note that

$$
\begin{aligned}
& \left.\mathbb{E}\left(\int_{0}^{t} \int_{0}^{\infty} \sum_{i} \alpha_{i} \mathbf{1}_{\left\{A_{i}\right\}} W(\mathrm{~d} s \mathrm{~d} a)\right)\left(\int_{0}^{t} \int_{-\infty}^{0} \sum_{j} \beta_{j} \mathbf{1}_{\{} B_{j}\right\} W(\mathrm{~d} a \mathrm{~d} s)\right) \\
& \quad=\sum_{i} \alpha_{i} \mathbb{E}\left(W\left(A_{i} \cap[0, t] \times[0, \infty)\right)\right) \sum_{j} \beta_{j} \mathbb{E}\left(W\left(B_{j} \cap[0, t] \times(-\infty, 0]\right)\right),
\end{aligned}
$$

where we have used the independent scattered property of Gaussian measures. This yields

$$
\mathbb{E}\left(I_{t}^{2}(f) I_{t}^{1}(g)\right)=\mathbb{E}\left(I_{t}^{1}(f) I_{t}^{2}(g)\right)=0 .
$$

Moreover,

$$
\begin{aligned}
\mathbb{E}\left(I_{t}^{1}(f) I_{t}^{1}(g)\right)+\mathbb{E}\left(I_{t}^{2}(f) I_{t}^{2}(g)\right)= & \int_{0}^{t} \int_{0}^{\infty}\left(f\left(\left(u_{s}^{0}\right)^{-1}(a)\right) g\left(\left(u_{s}^{0}\right)^{-1}(a)\right)\right) \mathrm{d} a \mathrm{~d} s \\
& +\int_{0}^{t} \int_{-\infty}^{0}\left(f\left(\left(u_{s}^{0}\right)^{-1}(a)\right) g\left(\left(u_{s}^{0}\right)^{-1}(a)\right)\right) \mathrm{d} a \mathrm{~d} s \\
= & \int_{0}^{t}\left\langle\mu_{s}^{0}, f g\right\rangle \mathrm{d} s,
\end{aligned}
$$

where we used $y:=\left(u_{s}^{0}\right)^{-1}(a)$ in the final step and observed the deterministic nature of the integrand. Notice that since $a \in \mathbb{R}$ then the integral with respect to $a$ has to be separated in this case; hence, we cannot use the properties of the Itô integral to directly derive the covariance as we did for the general SPDE.

Remark 1. As mentioned in the introduction, Schied [18] also proved the CLT for the SBM, however with a different setup. He considered the process

$$
\frac{1}{\beta}\left\langle f, X_{\beta^{2} t}-X_{0} P_{\beta^{2} t}\right\rangle
$$

where $f$ is a bounded Lipschitz continuous function in $\mathbb{R}^{d}, X_{t}$ is the SBM, and comparing with our process, $\beta=\sqrt{\varepsilon}$. After proving the tightness of this process in $\mathcal{C}\left([0,1] ; \mathbb{R}^{n}\right)$, he proved that the finite-dimensional marginal distributions converge weakly to those of an $n$-dimensional Wiener process $W$ with covariance

$$
\mathbb{E}\left(W_{t}^{i} W_{t}^{j}\right)=2 t \int f_{i} f_{j} \mathrm{~d} \mu,
$$

where $\mu$ is the initial measure of the SBM. This shows the weak convergence of the process to $W$ with covariance (16). We note that the covariance of our limit is different since our process depends on $\varepsilon$ as well as $t$. 


\subsection{Proof of Theorem 4}

From the SPDE (2) characterization, we see that in the case of the FVP,

$$
G\left(a, y, u_{s}^{\varepsilon}(y)\right)=\mathbf{1}_{\left\{a \leq u_{s}^{\varepsilon}(y)\right\}}-u_{s}^{\varepsilon}(y),
$$

which also satisfies (3) and (4) of $G\left(a, ., u_{s}^{\varepsilon}().\right)$ and so estimate (13) holds. Thus, we have

$$
\left\langle\tilde{Z}_{t}^{0}, f\right\rangle=-\int_{0}^{t} \int_{0}^{1} \int_{\mathbb{R}} P_{t-s}\left(\mathbf{1}_{\left\{a \leq u_{s}^{0}(y)\right\}}-u_{s}^{0}(y)\right) f^{\prime}(y) \mathrm{d} y W(\mathrm{~d} a \mathrm{~d} s)
$$

is a Gaussian process with 0 mean and covariance given below. For $f, g \in \&(\mathbb{R})$,

$$
\begin{aligned}
\operatorname{cov}\left(\left\langle\tilde{Z}_{t}^{0}, f\right\rangle,\left\langle\tilde{Z}_{t}^{0}, g\right\rangle\right) & \\
= & \int_{0}^{t} \int_{0}^{1} \int_{\mathbb{R}}\left(\mathbf{1}_{\left\{a \leq u_{s}^{0}(y)\right\}}-u_{s}^{0}\right) f^{\prime}(y) \mathrm{d} y \int_{\mathbb{R}}\left(\mathbf{1}_{\left\{a \leq u_{s}^{0}(r)\right\}}-u_{s}^{0}(r)\right) g^{\prime}(r) \mathrm{d} r \mathrm{~d} a \mathrm{~d} s \\
= & \int_{0}^{t} \int_{0}^{1}\left(\int_{\left(u_{s}^{0}\right)^{-1}(a)}^{\infty} f^{\prime}(y) \mathrm{d} y+\left\langle\mu_{s}^{0}, f\right\rangle\right)\left(\int_{\left(u_{s}^{0}\right)^{-1}(a)}^{\infty} g^{\prime}(r) \mathrm{d} r+\left\langle\mu_{s}^{0}, g\right\rangle\right) \mathrm{d} a \mathrm{~d} s \\
= & \int_{0}^{t} \int_{0}^{1}\left(-f\left(\left(u_{s}^{0}\right)^{-1}(a)\right)+\left\langle\mu_{s}^{0}, f\right\rangle\right)\left(-g\left(\left(u_{s}^{0}\right)^{-1}(a)\right)+\left\langle\mu_{s}^{0}, g\right\rangle\right) \mathrm{d} a \mathrm{~d} s \\
= & \int_{0}^{t} \int_{0}^{1}\left(-f(y)+\left\langle\mu_{s}^{0}, f\right\rangle\right)\left(-g(y)+\left\langle\mu_{s}^{0}, g\right\rangle\right) \mu_{s}^{0}(\mathrm{~d} y) \mathrm{d} s \\
= & \int_{0}^{t} \int_{0}^{1} f(y) g(y) \mu_{s}^{0}(\mathrm{~d} y) \mathrm{d} s-\int_{0}^{t} \int_{0}^{1}\left\langle\mu_{s}^{0}, f\right\rangle g(y) \mu_{s}^{0}(\mathrm{~d} y) \mathrm{d} s \\
& -\int_{0}^{t} \int_{0}^{1} f(y)\left\langle\mu_{s}^{0}, g\right\rangle \mu_{s}^{0}(\mathrm{~d} y) \mathrm{d} s+\int_{0}^{t}\left\langle\mu_{s}^{0}, f\right\rangle\left\langle\mu_{s}^{0}, g\right\rangle \mathrm{d} s .
\end{aligned}
$$

Therefore, the CLT for the FVP is achieved.

\section{Acknowledgements}

I would like to thank my Ph.D. advisor Dr Jie Xiong for giving me useful ideas for this paper. I am also thankful to Dr Kei Kobayashi for pointing out the independent scattered property of Gaussian measures that was used in the case of the SBM. In addition, my gratitude goes to the anonymous referee who gave me good comments on how to improve the paper.

\section{References}

[1] Billingsley, P. (1968). Convergence of Probability Measures. John Wiley, New York.

[2] Dawson, D. A. And Feng, S. (1998). Large deviations for the Fleming-Viot process with neutral mutation and selection. Stoch. Process. Appl. 77, 207-232. (Correction: 88 (2000), 175.)

[3] Etheridge, A. M. (2000). An Introduction to Superprocesses (Univ. Lecture Ser. 20). American Mathematical Society, Providence, RI.

[4] Fatheddin, P. and Xiong, J. (2015). Large deviation principle for some measure-valued processes. Stoch. Proc. Appl. 125, 970-993.

[5] Fatheddin, P. And Xiong, J. (2015). Moderate deviation principle for a class of SPDEs. To Appear in J. Appl. Prob.

[6] Feng, S. And Xiong, J. (2002). Large deviations and quasi-potential of a Fleming-Viot process. Electron. Commun. Prob. 7, 13-25.

[7] Hong, W. (1998). Central limit theorem for the occupation time of catalytic super-Brownian motion. Chinese Sci. Bull. 43, 2035-2040. 
[8] Hong, W. (2002). Longtime behavior for the occupation time process of a super-Brownian motion with random immigration. Stoch. Process. Appl.. 102, 43-62.

[9] Hong, W. (2005). Quenched mean limit theorems for the super-Brownian motion with super-Brownian immigration. Infin. Dimens. Anal. Quantum Prob. Relat. Top. 8, 383-396.

[10] Hong, W.-M. AND LI, Z.-H (1999). A central limit theorem for super-Brownian motion with super-Brownian immigration. J. Appl. Prob. 36, 1218-1224.

[11] Hong, W. And Li, Z. (2001). Fluctuations of a super-Brownian motion with randomly controlled immigration. Statist. Prob. Lett. 51, 285-291.

[12] Hong, W. ANd Wang, Z. (2000). Immigration process in catalytic medium. Sci. China Ser. A 43, 59-64.

[13] Hong, W. And Zeitouni, O. (2007). A quenched CLT for super-Brownian motion with random immigration. J. Theoret. Prob. 20, 807-820.

[14] Iscoe, I. (1986). A weighted occupation time for a class of measure-valued branching processes. Prob. Theory Relat. Fields 71, 85-116.

[15] LeE, T.-Y AND Remillard, B. (1995). Large deviations for the three-dimensional super-Brownian motion. Ann. Prob. 23, 1755-1771.

[16] LI, Z. (1999). Some central limit theorems for super Brownian motion. Acta Math. Sci. 19, 121-126.

[17] Mitoma, I. (1983). Tightness of probabilities on $\mathcal{C}\left([0,1] ; \digamma^{\prime}\right)$ and $D\left([0,1] ; \digamma^{\prime}\right)$. Ann. Prob. 11, 989-999.

[18] Schied, A. (1997). Moderate deviations and functional LIL for super-Brownian motion. Stoch. Process. Appl. 72, 11-25.

[19] Whitт, W. (2002). Stochastic-Process Limits: An Introduction to Stochastic-Process Limits and Their Application to Queues. Springer, New York.

[20] XIong, J. (2013). Super-Brownian motion as the unique strong solution to an SPDE. Ann. Prob. 41, 1030-1054.

[21] Zhang, M. (2008). Some scaled limit theorems for an immigration super-Brownian motion. Sci. China Ser. A 51, 203-214.

[22] Zhang, M. (2009). On the weak convergence of super-Brownian motion with immigration. Sci. China Ser. A 52, 1875-1886. 